\title{
URAN, A DECAMETER BAND VLBI SYSTEM
}

\author{
S. J. Braude, L. N. Litvinenko, A. V. Megn \\ Institute of Radio Astronomy \\ Kharkov, U.S.S.R.
}

Until recently, regular interferometric measurements were not performed at decameter wavelengths. The reasons are well known, i.e., effects of the ionosphere and the interplanetary plasma, the inherently high level of interference and of the galactic background, and the necessity to have very large antennas. Yet, because of the considerable interest in such observations, the Ukrainian SSR Academy of Sciences has started a project aimed at the development and construction of a system of decameter band interferometers (project URAN, from the "Ukrainian Radio Interferometers of Academy of Sciences (Nauk)"). By 1989, the system will involve four interferometers with baseline lengths between 40 and $900 \mathrm{~km}$. The basic instrument of all these is the N-S arm of the large UTR-2 radio telescope operated by the Institute of Radio Astronomy in Kharkov. The antenna is an electrically controlled phased array of broad band dipoles (total length about $1800 \mathrm{~m}$ ). At the other sites, the antennas are smaller phased arrays (maximum length about $230 \mathrm{~m}$ ) consisting of crossed dipoles.

The shortest baseline is $42.2 \mathrm{~km}$, achieved with the URAN-1 interferometer near Kharkov. It is oriented along the $\mathrm{E}-\mathrm{W}$ direction and has been used over a decade for connected element interferometry at 25,20 and 16.7 MHz. New techniques for measuring the magnitude $\gamma$ of the visibility function have been developed, with an account of the special observation conditions at these frequencies. $\gamma$ has been measured for about 30 sources of radio emission such as supernova remnants, radio galaxies and quasars. Some of the galaxies and quasistellar objects (e.g., 3C196 or 3C84A) have shown extended components of tens seconds of arc in size, characterized by "steep" power law spectra, that do not seem to have been observed before at higher frequencies. Compact components with steep spectra have been detected and investigated in supernova remnants (3C144 and 3C461). Measurements of the angular structure of the radiation from extended sources like radio galaxies or unidentified objects (e.g., $3 \mathrm{C} 123$ or $3 \mathrm{C} 134$ ) are in agreement with the meter and decimeter wavelength data.

Preliminary observations of 3C144, 3C196 and 3C461 were performed in 1986/87 with the new VLB interferometer URAN-3 (baseline length $616 \mathrm{~km}$ ). We have found that high quality VLB records can be obtained at decameter wavelengths in autumn and winter during the night time, and $\gamma$ can be estimated to within 5 or 10 percent. 